\title{
ENTORNOS MPLS Y ATM PARA EL TRANSPORTE DE VOZ SOBRE INTERNET
}

\section{MPLS AND ATM ENVIRONMENTS FOR CARRYING VOICE OVER THE INTERNET}

\begin{abstract}
The present article shows the results obtained from a Quality-ofService-based study conducted over IP networks when carrying delay-sensitive traffic, namely VoIP over ATM and MPLS. An analysis of different network topologies is provided. The characteristics that pertain to each topology allow an interesting evaluation of variables such as delay and bandwidth.
\end{abstract}

Key words: ATM, CR-LDP, MPLS, RSVP-TE, LSP, FEC.

\section{RESUMEN}

Este artículo presenta los resultados obtenidos en el estudio de la calidad de servicio sobre redes IP en tráficos sensibles al retardo como la VoIP sobre la tecnología ATM y MPLS. Se analizaran diferentes topologías con características propias que permitirán evaluar valores tales como el retardo y el ancho de banda.

Palabras claves: ATM, CR-LDP, MPLS, RSVP-TE, LSP, FEC.

\section{Nancy Yaneth Gelvez García}

Magister en Ciencias de la Información y las Comunicaciones

Docente planta de la Universidad Distrital Francisco José de Caldas Bogotá, Colombia

nayagarcia@udistrital.edu.co

\section{Luis Fernando Pedraza}

Magister en Ciencias de la Información y las Comunicaciones

Docente planta de la Universidad Distrital Francisco José de Caldas Bogotá, Colombia

lfpedrazam@udistrital.edu.co

Tipo: Artículo de investigación

Fecha de Recepción: Febrero 27 de 2012

Fecha de Aceptación: Mayo 2 de 2012 


\section{INTRODUCCIÓN}

La convergencia de servicios en la red internet implica un aumento importante en el tráfico circundante; esto hace que la networking deba contar con diferentes estructuras lógicas que le permitan soportar múltiples flujos de información (susceptibles y no susceptibles a los retardos). Dentro de las aplicaciones que más exigencias demanda, está la voz que requiere de la reserva de ciertos parámetros para que el cliente tenga una experiencia placentera con el servicio prestado por el proveedor de servicios de internet. Todo ello se traduce en la utilización de una plataforma hardware y software adecuada que no solo soporte el tráfico de voz, si no otro tipos de transmisiones como multimedia. Esto ha hecho que frame relay haya dejado de ser llamativa, teniéndose que utilizar otro tipo de estándares que soporten varios flujos donde los requerimientos difieren drásticamente. A partir de este análisis surge la necesidad de establecer si MPLS o ATM es la más adecuada para soportar el envió y retransmisión de voz.

\section{MARCO TEÓRICO}

De V/IP no se escucho hablar si no hasta la década de los 90 cuando un pequeño grupo de investigación en Israel comenzó a trabajar en un nuevo concepto de comunicación entre PC's directamente. Con el paso de los años se fué fortaleciendo el concepto de la transferencia de la señal de Voz en paquetes que se lograran transmitir a través de la súper-autopista de las comunicaciones. Los grandes fabricantes vieron un potencial en esta tecnología y comenzaron a ofertar las primeras plataformas para VozIP y ATA / gateways [1] aumentando el tráfico en la red.

A comienzos del nuevo siglo se crea Asterisk [2], una central telefónica / conmutador basada en linux, que podía ser instalada en una PC y prestar el servicio de VoIP a partir de un código abierto ofreciendo un potencial de crecimiento para hogares o pequeñas empresas que quisieran implementar esta tecnología. Tres años después se crea el softphone gratuito más popular para PC, skype [3], cuya característica fundamental es la de permitir la comunicación a través de todos los firewalls y routers de las redes convirtiéndose en el referente para las aplicaciones VozIP. Hoy en día este tipo de servicios se ha convertido en una exigencia por parte de los usuarios lo que se traduce para los ISPs en la búsqueda y evaluación de tecnologías de redes que permitan establecer conexiones uniformes que permitan conversaciones legibles de extremo a extremo.

Por ser una aplicación sensible al retardo se debe asegurar una mínima latencia para que haya una buena fluidez en la conversación; se considera aceptable un valor por debajo de los 150 ms. Además existen otras variables que deben ser tenidas en cuenta entre las que se encuentran (ver tabla 1):

- Priorización de paquetes para reducir latencia.

- Supresión de silencios.

- Compresión de cabeceras.

- Soporte de IPv6

Tabla 1. Calidad de funcionamiento de diversas aplicaciones [5].

\begin{tabular}{|l|l|l|c|c|c|c|}
\hline \multicolumn{4}{|c|}{} & \multicolumn{3}{c|}{$\begin{array}{c}\text { Parámetros clave y valores de objetivo para la } \\
\text { calidad de funcionamiento }\end{array}$} \\
\hline \multicolumn{1}{|c|}{ Medio } & Aplicación & $\begin{array}{c}\text { Grado de } \\
\text { simetría }\end{array}$ & $\begin{array}{c}\text { Velocidad de } \\
\text { datos típicas }\end{array}$ & $\begin{array}{c}\text { Tiempo de transmisión } \\
\text { en un sentido }\end{array}$ & $\begin{array}{c}\text { Variación } \\
\text { de retardos }\end{array}$ & $\begin{array}{c}\text { Perdida de } \\
\text { información }\end{array}$ \\
\hline Audio & $\begin{array}{l}\text { Audio en } \\
\text { tiempo real }\end{array}$ & $\begin{array}{l}\text { Principalmente } \\
\text { en un sentido }\end{array}$ & $4-32 \mathrm{Kbits} / \mathrm{s}$ & $<10 \mathrm{~s}$ & $<<1 \mathrm{~ms}$ & PLR<3\% \\
\hline Video & $\begin{array}{l}\text { Video } \\
\text { teléfono }\end{array}$ & Dos sentidos & $\begin{array}{l}16-384 \\
\text { Kbits/s }\end{array}$ & $<150 \mathrm{~ms}$ & & PLR $<1 \%$ \\
\hline $\begin{array}{l}\text { Correo } \\
\text { electrónico }\end{array}$ & $\begin{array}{l}\text { Acceso al } \\
\text { servidor }\end{array}$ & $\begin{array}{l}\text { Principalmente } \\
\text { un sentido }\end{array}$ & $<10 \mathrm{~Kb}$ & $<4 \mathrm{~s}$ & N.A & Nula \\
\hline
\end{tabular}


El cumplimiento de las especificaciones estandarizadas por la ITU-T y visualizadas en la tabla anterior, sólo se logran siempre y cuando la networking cuenta con algoritmos que convergan rápidamente y se adapten a los cambios reservando recursos necesarios exigidos por la aplicación específica.

Dentro de los protocolos más robustos para el soporte eficiente de voz sobre IP esta el modo de transmisión asíncrona (ATM) y la conmutación de etiquetas multiprotocolo (MPLS). No obstante es importante para los ISPs tener un referente que determine cuál es el más robusto y adecuado en el transporte de voz. Por tal motivo a continuación se presenta una serie de simulaciones que muestran los resultados encontrados al evaluar cada tecnología cuando dentro de la estructura existe un cuello de botella y además aparecen eventos inesperados como la necesidad de reencaminar los datos por caminos alternativos.

\section{SIMULACIÓN Y ANÁLISIS DE EVENTOS}

Para el desarrollo de la investigación se trabajo sobre las topologías mostradas en la Fig. 1 y 3. El software utilizado para ejecutar y analizar las estructuras propuestas fue opnet (versión académica) [6], donde es importante destacar que el consumo de recursos de la máquina y el tiempo de simulación de la red es alto, por tal motivo los eventos se generaron para espacios de tiempo relativamente cortos (en promedio 7 minutos).

\subsection{Topología "cuello de botella"}

En la Fig. 1, se observa el esquema utilizado para el primer caso, donde es evidente el cuello de botella en el nodo router_core1.

Todos los enlaces son OC3 y poseen un ancho de banda de 148.61Mbps. Cada encaminador (router1, router2, router3, router4, router core1, router_core2, router_core3, router core4) soporta ATM o MPLS (este último asociado con CR-LDP o RSVP-TE) dependiendo de la estructura sobre la que se esté trabajando. Los Nodos que aparecen bautizados como
"Red1, Red2, Red3" hacen las veces de servidores y son generadores de tráfico del tipo FTP, e-mail, video-streming y voz, mientras que "red4, red5", son PCs convencionales que hacen las funciones de sumideros o receptores del flujo. Estos dispositivos finales tienen implementado Ethernet de 100Mbps.

La configuración de parámetros de clases de servicio en ATM (UBR o CBR) se implementó de acuerdo al tipo de tráfico que circule por el nodo de ingreso a la nube ATM (router1 o router2 o router3), para el caso de MPLS según la FEC. La política de encolamiento utilizado fue priority queuing basado en DSCP.

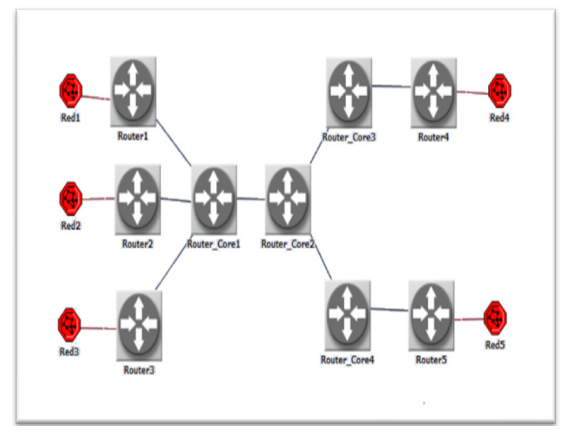

Fig. 1. Topología cuello de botella.

Como se puede ver en la Fig. 1 el recurso por el que se va a competir es el Ancho de Banda existente entre "router_core1"y "router_core2" y lo que se pretende obtener es la variación del retardo generado por cada técnica a fin de poder establecer cual es la más eficiente.

La Fig. 2 y tabla 2 condensa la variación del retardo encontrado para la voz.

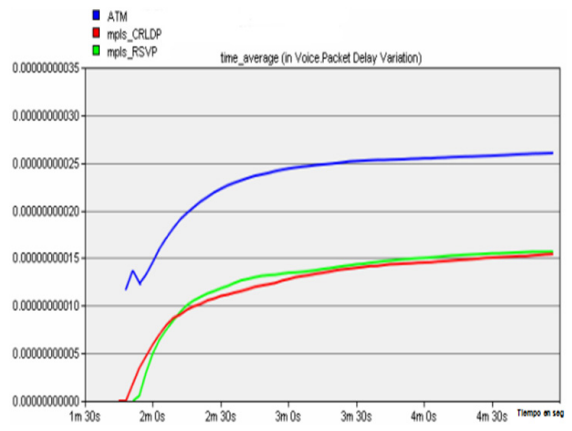

Fig. 2. Variación del retardo de ATM, MPLS CR-LDP y MPLS RSVP-TE. 
Tabla 2. Valores obtenidos con ATM y MPLS.

\begin{tabular}{|c|c|c|c|c|c|}
\hline & $\begin{array}{c}\text { Paquetes } \\
\text { enviados }\end{array}$ & $\begin{array}{c}\text { Paquetes } \\
\text { recibidos }\end{array}$ & \multicolumn{2}{|c|}{$\begin{array}{c}\text { Delay variation } \\
{[\mathrm{s}]}\end{array}$} & \multirow{2}{*}{$\begin{array}{c}\text { Delay end to end } \\
{[\mathrm{s}]}\end{array}$} \\
\hline & \multicolumn{2}{|c|}{$[$ Paquetes $/ \mathrm{s}]$} & Min & Max & \\
\hline ATM & 47.107 & 47.107 & $1.17 \times 10^{-10}$ & $2.60 \times 10^{-10}$ & 0.0717 \\
\hline CR-LDP & 45.707 & 41.643 & $5.15 \times 10^{-20}$ & $1.54 \times 10^{-10}$ & 0.0656 \\
\hline RSVP-TE & 50.303 & 49.850 & 0 & $1.56 \times 10^{-10}$ & 0.0650 \\
\hline
\end{tabular}

Concretamente, se encuentra que ATM tiene un menor desempeño que MPLS asociado con los protocolos de señalización CR-LDP y RSVP-TE (el cual corresponde a un $40 \%$ en sus valores máximos), esto debido al tratamiento que tiene ATM a los diferentes flujos de tráfico en su nivel de aplicación, donde existe un mayor gasto de procesamiento para el envío de cualquier tipo de datos en los nodos, ya que cada uno de estos debe hacer un proceso de lectura de encabezado IP y decidir el siguiente salto, lo cual no ocurre en MPLS, pues este tiene un gasto mínimo de procesamiento en los LSR (router_core1 y router core2) al tener que analizar únicamente una etiqueta.

La mejor calidad de servicio prestada por MPLS es producida gracias a la reserva de recursos establecida por los protocolos RSVP-TE y CRLDP. Adicionalmente se puede concluir que no existe una diferencia muy marcada en cuanto a la variación del retardo generado por estos tipos de señalización, lo que se traduce en un comportamiento muy similar.

En cuanto a la perdida de paquetes, ATM muestra un excelente comportamiento debido a que todos los paquetes enviados fueron recibidos. Por otro lado, MPLS si presenta un descarte de paquetes que aunque es alta, al verla desde los valores de ATM, no supera el estándar del 3\% presentada en la tabla 1, que indica el nivel de tolerancia soportado por aplicaciones como la voz.

\subsection{Topología "de re-enrutamiento"}

La Fig. 3, muestra que la topología de trabajo parte de la utilizada en el análisis anterior a la que se le incluye el nodo adicional "router core5" para crear una ruta redundante a la inicialmente establecida, adicionalmente se reduce el ancho de banda disponible entre el router_core 1 y el router_core 2 a un E1 y de esta forma poner a prueba la eficiencia de ATM y MPLS para aplicar re-encaminamiento por la ruta: router_core1, router_core5, router_core2 la cual posee el ancho de banda necesario (OC3) para soportar el flujo de voz.

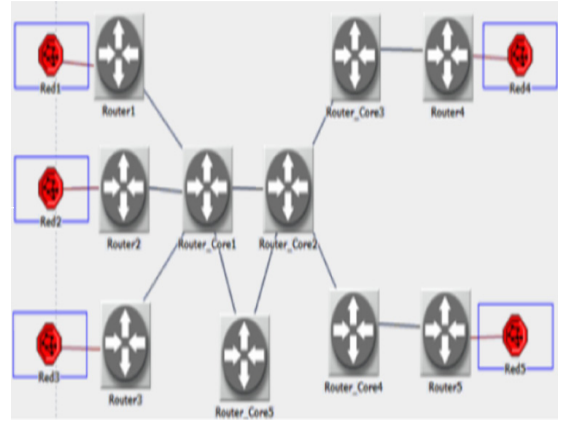

Fig. 3. Topología re-enrutamiento.

Los resultados encontrados para el caso ATM (utilizando como protocolo de routing OSPF) se plasman en la Fig. 4 y 5. Se observa que a pesar de la necesidad de buscar rutas alternas, la capacidad de entrega no se vio alterada, en cambio el retardo aumento considerablemente (unas 4 veces en comparación a la topología 1 trabajada anteriormente) debido al esfuerzo que debe hacer ATM para decidir por donde debe re-direccionar los paquetes.

El comportamiento obtenido con MPLS+RSVPTE y MPLS+CR-LDP aparece en las Fig. 6 y 7. De allí se deduce que la información de envió y recepción en cada caso es casi idéntico, lo que demuestra la garantía de ancho de banda. 


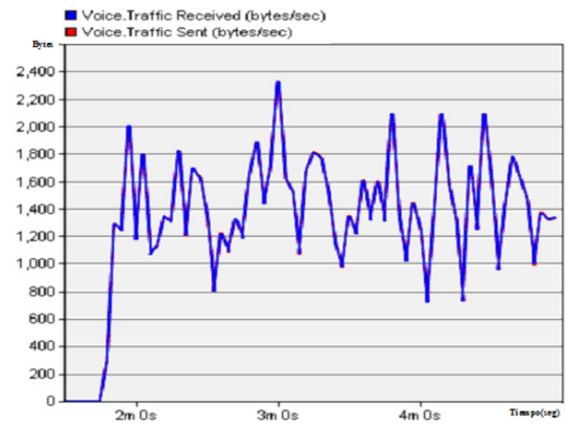

Fig. 4. Flujo de voz IP enviado y recibido por ATM.

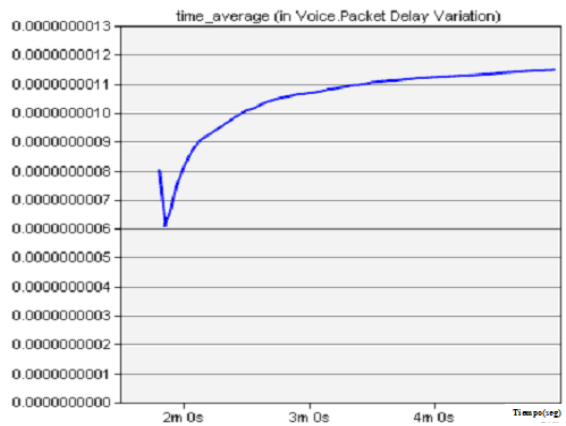

Fig. 5. Jitter generado para la voz en ATM.

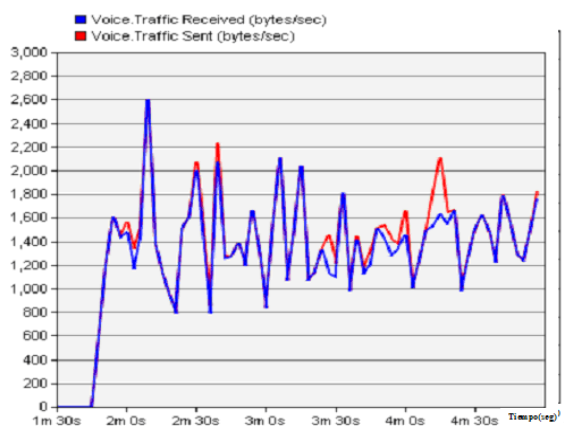

Fig. 6. Tráfico de voz con CR-LDP.

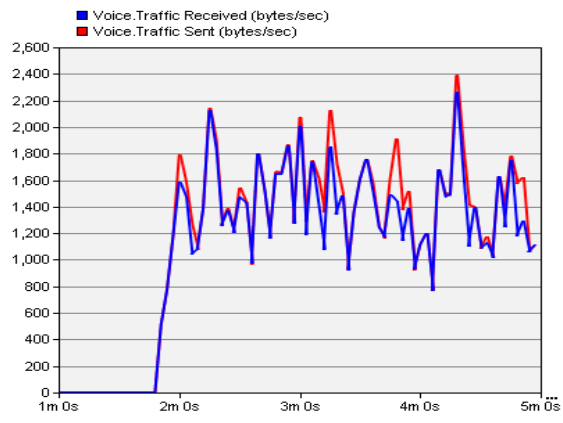

Fig. 7. Tráfico de voz con RSVP-TE.

En cuanto al jitter (Fig. 8), resulta evidente que poseen diferentes conductas, pues CR-
LDP se comporta mejor en toda la simulación, ya que llega a un máximo de $9.82 \times 10^{-10}$ en comparación a RSVP-TE con $1.03 \times 10^{-9}$ que es un $4.6 \%$ más.

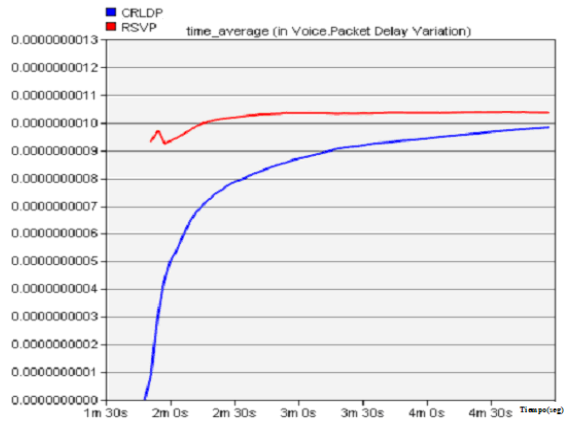

Fig. 8. Jitter en MPLS.

Finalmente, a manera de comparación de los tres métodos se muestra (Fig. 9) dentro de un mismo par de ejes los resultados encontrados en cada caso.

El mejor desempeño lo tiene MPLS soportado en sus dos protocolos de señalizaciones en comparación a ATM, donde CR-LDP tiene una diferencia de $0.18 \times 10^{-10} \mathrm{~s}$, con relación a ATM y de $0.05 \times 10^{-10} \mathrm{~s}$ con respecto RSVP-TE. De este resultado se infiere que CR-LDP le está dando mayor prioridad al tráfico de Voz IP.

Al analizar las estadísticas de perdida de paquetes se puede ver que ATM tiene un mejor comportamiento ya que presenta el $0,006 \%$ de perdidas, en contraste a MPLS que en esta topología tiene el $4,1 \%$ si se asocia con RSVPTE y $8,7 \%$ si se combina con CR-LDP (tabla 3 ).

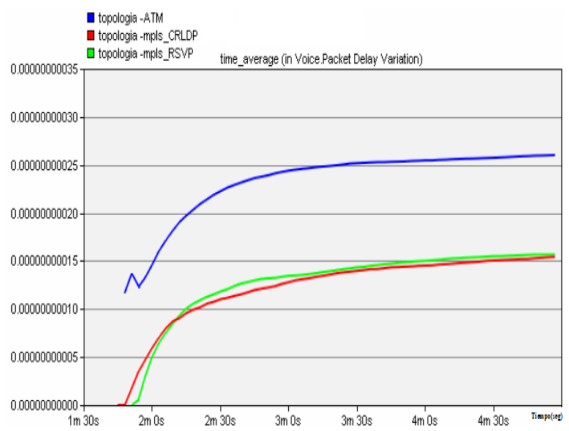

Fig. 9. Tiempo de retardo de Voz para las tres tecnologías. 
Tabla 3. Comparación entre ATM y MPLS para voz.

\begin{tabular}{|c|c|c|c|c|c|}
\hline & $\begin{array}{c}\text { Paquetes } \\
\text { enviados }\end{array}$ & $\begin{array}{c}\text { Paquetes } \\
\text { recibidos }\end{array}$ & \multicolumn{2}{|c|}{$\begin{array}{c}\text { Delay variation } \\
{[\mathrm{s}]}\end{array}$} & \multirow{2}{*}{$\begin{array}{c}\text { Delay end to end } \\
{[\mathrm{s}]}\end{array}$} \\
\hline & \multicolumn{2}{|c|}{$[$ Paquetes/s] } & Min & Max & \\
\hline ATM & 47.107 & 47.107 & $1.17 \times 10^{-10}$ & $2.60 \times 10^{-10}$ & 0.0717 \\
\hline CR-LDP & 41.643 & 45.707 & $5.15 \times 10^{-20}$ & $1.54 \times 10^{-10}$ & 0.0656 \\
\hline RSVP-TE & 49.850 & 50.303 & 0 & $1.56 \times 10^{-10}$ & 0.0650 \\
\hline
\end{tabular}

En topologías con cuellos de botella, MPLS es superior en cuanto a la variación del retardo, el cual se va a ver reflejado en una mejor QoS hacia el usuario final en particular para paquetes de voz. Por otro lado, ATM tiene un mejor comportamiento con relación a los paquetes descartados de aplicaciones que

toleran errores, pero con un sacrificio en el retardo.

La superioridad de MPLS mostrada en esta investigación frente a ATM confirma el buen trabajo realizado por los diseñadores para resolver los inconvenientes que se presentan en las actuales redes, esto también se puede soportar en los diversos estudios que se encuentran publicados y que de alguna una otra forma ayudan a un mejor entendimiento de la filosofía inmersa en la conmutación de etiquetas multiprotocolo.

\section{Referencias Bibliográficas}

[1] [En línea], consultado en Julio 10 del 2010, disponible en: http://www. cisco.com/web/solutions/smb/espanol/ productos/voz_conferencias/gateways _ ata_voz_pequenas_empresas.html.

[2] [En línea], consultado en Junio 21 del 2010, disponible en: http://www.asterisk. org/.

[3] [En línea], consultado en Septiembre 11 del 2010, disponible en: http://www. skype.com/intl/es/features/.

[4] ITU-T G.113. Transmission systems and media, digital systems and networks. [En línea], consultado en Agosto 17 del 2010, disponible en: http://www.itu.int/rec/TREC-G.113-200711-I.

[5] ITU-T. G1010. Sistemas y medios de transmisión, sistemas y redes digitales. [En línea], consultado en Octubre 18 de 2010, disponible en: http://www.itu.int/ rec/T-REC-G.1010-200111-I/es.

[6] [En línea], consultado en Abril 7 del 2010, disponible en: www.opnet.com.

[7] E. William, F. Clarence, Deploying IP and MPLS QoS for multiservice networks: Theory \& practice, editorial Morgan Kauffman, pp: 24, 38-59, 87-99, 2007.

[8] M. Ina, L. Julian, MPLS-Enabled applications: emerging developments and new technologies, editorial Wiley, pp: $37-$ 48, 2005. 